\title{
Recurrent waist tumor subsequent to percutaneous nephrolithotomy: A case report
}

\author{
SHUSUAN JIANG ${ }^{1 *}$, SULAI LIU $^{1,2^{*}}$, PINGPING TAN $^{3}$, TIEYONG SONG $^{1}$, YU XIE $^{1}$, \\ FUHUA ZENG $^{1}$, ZHIZHONG LIU ${ }^{1}$, WEIQING HAN ${ }^{1}$, ZENGNAN MO ${ }^{4}$ and LIN QI ${ }^{2}$ \\ ${ }^{1}$ Department of Urology, The Affiliated Cancer Hospital of Xiangya School of Medicine, \\ Central South University, Changsha, Hunan 410013; ${ }^{2}$ Department of Urology, Xiangya Hospital, Central South University, \\ Changsha, Hunan 410008; ${ }^{3}$ Department of Pathology, The Affiliated Cancer Hospital of Xiangya School of Medicine, \\ Central South University, Changsha, Hunan 410013; ${ }^{4}$ Institute of Urology and Nephrology, \\ The First Affiliated Hospital of Guangxi Medical University, Nanning, Guangxi 530021, P.R. China
}

Received June 25, 2014; Accepted March 24, 2015

DOI: $10.3892 / \mathrm{ol} .2015 .3175$

\begin{abstract}
The present study reports the case of a 39-year old male patient with a recurrent waist tumor that occurred subsequent to percutaneous nephrolithotomy (PCNL). The patient initially underwent PCNL for the management of right calculus of the kidney. Six years later, the patient underwent local mass resection for a tumor at the waist, which was subsequently diagnosed as adenocarcinoma. However, seven months subsequent to local resection, the patient presented to the Affiliated Cancer Hospital of Xiangya Medical School with a one-month history of a recurrent tumor located at the right waist. Physical examination identified no visible skin lesions; however, a palpable hard nodule was present over the right waist. Imaging studies, consisting of computed tomography (CT) and positron emission tomography-CT, indicated no additional metastases. Therefore, the patient underwent local mass resection of the waist tumor. Subsequent histological examination determined a diagnosis of metastatic adenocarcinoma. Considering the previously conducted PCNL surgery and the diagnosis, it is proposed that the recurrent waist tumor originated from renal cell carcinoma (RCC), also termed renal adenocarcinoma. However, no evidence of the original RCC tumor was identified. Therefore, the selection of an effective treatment strategy was challenging.
\end{abstract}

Correspondence to: Professor Weiqing Han, Department of Urology, The Affiliated Cancer Hospital of Xiangya School of Medicine, Central South University, 283 Tongzipo Road, Changsha, Hunan 410013, P.R. China

E-mail:hwq01@vip.163.com

${ }^{*}$ Contributed equally

Key words: percutaneous nephrolithotomy, renal cell carcinoma, waist tumor

\section{Introduction}

Employing percutaneous nephrolithotomy (PCNL) surgery for the management of large renal stones has been proven to be effective and is an accepted treatment strategy worldwide (1-3). The good functional results and associated decrease in surgical morbidity have encouraged the use of PCNL, as opposed to open pyelolithotomy or nephrolithotomy, even in complicated cases, such as complete staghorn stones associated with chronic infection and previous procedures $(4,5)$.

During the treatment of such challenging cases, the possibility of an underlying renal cell carcinoma (RCC) or urothelial carcinoma requires consideration (6,7). Preoperative diagnosis of such lesions may be challenging due to the presence of a stone and the associated inflammatory process. PCNL enables direct investigation of the renal pelvis. However, it is difficult for PCNL to identify RCC during surgery. The current study reports the case of a 39-year-old male patient exhibiting a recurrent waist tumor subsequent to PCNL that was determined to be metastatic adenocarcinoma. To the best of our knowledge, there are no previous reports of recurrent metastatic adenocarcinoma of the waist following PCNL in the literature. Notably, no tumor was detected in the right kidney using B-ultrasound, computed tomography (CT) or positron emission tomography (PET)-CT. Therefore, the current study is unique.

\section{Case report}

In April 2014, a 39-year-old man presented to the Affiliated Cancer Hospital of Xiangya Medical School with a one-month history of a recurrent right waist tumor. Examination of the patient's medical history revealed that PCNL had been performed for right calculus of the kidney at Xiangya Hospital, Central South University (Changsha, China) in June 2008. Following PCNL, the patient did not receive post-operative therapy and was in a good general condition. However, seven months prior to admission to our clinic, a soft tissue mass was noted at the previous PCNL tract site. The patient underwent local mass resection for the right waist 
tumor and immunohistochemical examination of the resected specimen revealed poorly differentiated metastatic adenocarcinoma (Fig. 1A and B); Mitotic figures in high-power fields were observed, as well as intercellular bridges and focal keratinization. Furthermore, tumor cells exhibited strong positive staining for Vim and epithelial membrane antigen. Considering that the patient had previously undergone PCNL, it was proposed that the metastatic tumor had originated from RCC. The patient did not receive any post-operative adjuvant therapy and was in a good general condition. Upon presentation at our clinic, the patient underwent a physical examination. The examination revealed a well-healed right flank scar and a $4 \times 7-\mathrm{cm}$ tumor under the scar exhibiting a painless, indurated, firm and immobile nodule (Fig. 2C and D). Upon excretory urography, the left kidney was determined to be poorly functioning, demonstrating atrophy, and right moderate hydronephrosis with large staghorn stones was diagnosed (Fig. 2A). Additionally, a CT scan identified the soft tissue mass (Fig. 2B and C) and a PET-CT scan revealed an increased radioactivity concentration at the tumor site in the right waist (Fig. 2D). There was no evidence of additional tumor spread or lymph node involvement. Notably, there were also no abnormal findings on the right kidney, excluding recurrent calculi. In consideration of the aforementioned findings, a local mass resection of the waist tumor was performed. Subsequent histopathological examination revealed a poorly differentiated adenocarcinoma that conformed to the characteristics of neoplasm recurrence (Fig. 3). Post-operatively, the patient received systemic chemotherapy, consisting of the administration of gemcitabine $\left(1000 \mathrm{mg} / \mathrm{m}^{2}\right.$; days 1 and 8; Q3W) plus carboplatin (area under curve, 5; day 1, Q3W), for the treatment of the metastatic tumor, and was disease-free at the most recent three-month follow-up. However, notably, the primary tumor was not identified by imaging and clinical analysis. Considering that the left kidney was poorly functioning and demonstrated atrophy, right moderate hydronephrosis with large staghorn stones was identified.

In accordance with the regulations of the Human Investigation Committee of the Central South University (Changsha, China), written informed consent was obtained from the patient for publication of the current report and any accompanying images.

\section{Discussion}

Following its introduction in 1976, PCNL has become the preferred surgical procedure for the treatment of patients with large and complex calculi of the kidneys (6). For example, according to the 2005 American Urological Association clinical guidelines, PCNL is the recommended first-line treatment strategy for calculi with surface area $>500 \mathrm{~mm}^{2}$ (7). Although PCNL can result in a good stone-free rate of $78-95 \%$ (8), it has been associated with significant complications, such as loss of a kidney (due to the necessity for complete resection), urinary leakage, uncontrolled hemorrhage, sepsis, tumor seeding, injury to the collecting system and surrounding viscera, or mortality $(8,9)$. Therefore, undergoing PCNL surgery poses a significant risk for patients, particularly those exhibiting occult RCC.

To the best of our knowledge, no cases of RCC extension along a PCNL tract have been reported in the literature in recent years. The current study reports the case of a recurrent
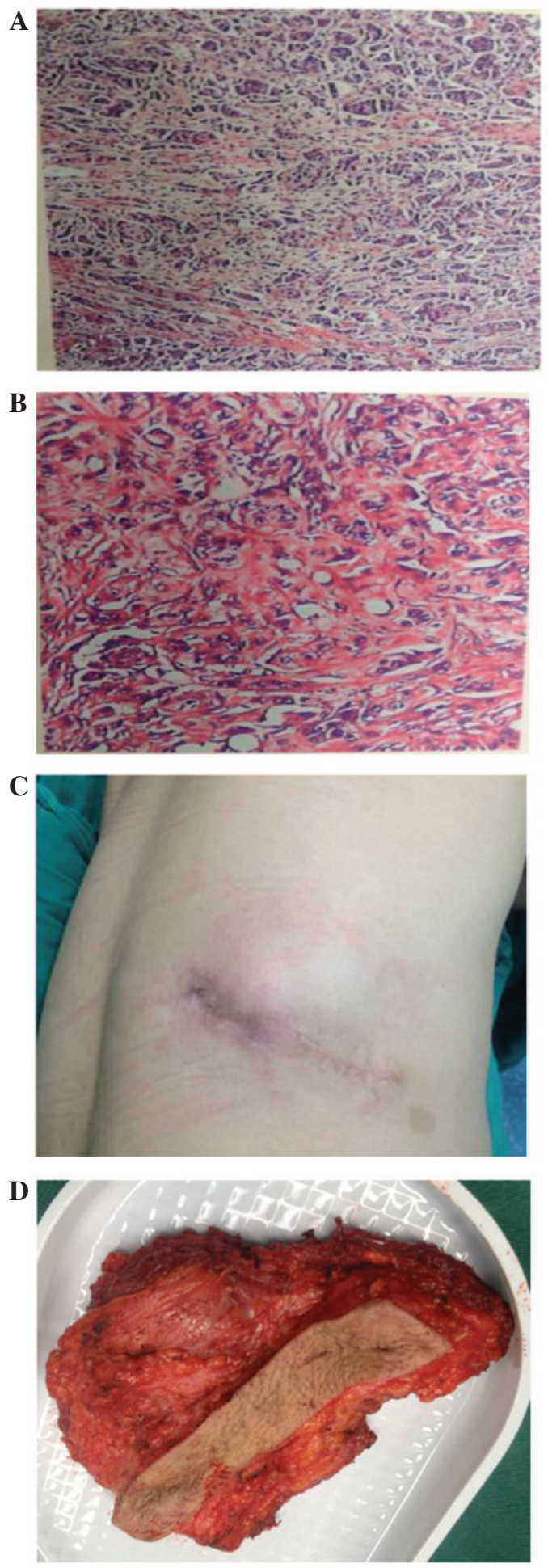

Figure 1. (A and B) Immunohistochemical findings of the initial surgical resection following percutaneous nephrolithotomy (PCNL), indicating that the tumor origin was renal cell carcinoma: (A) Vim- and (B) epithelial membrane antigen-positive staining in the tumor cells (magnification, x100). (C) A soft tissue mass at the PCNL surgical incision site. (D) Gross specimen revealing a large mass associated with the PCNL tract.

right waist tumor extending along a PCNL tract that was diagnosed as RCC of suspicious origin upon pathological analysis of the tumor specimen. However, prior to intervention, intravenous pyelography, CT and PET-CT scans were performed without presenting evidence of the suspected RCC. 
A
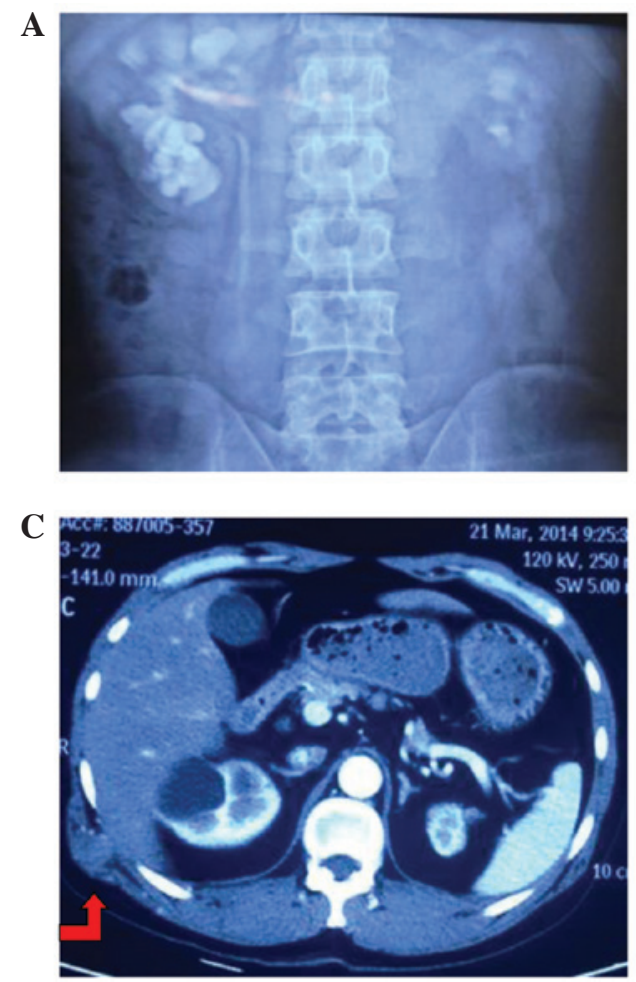
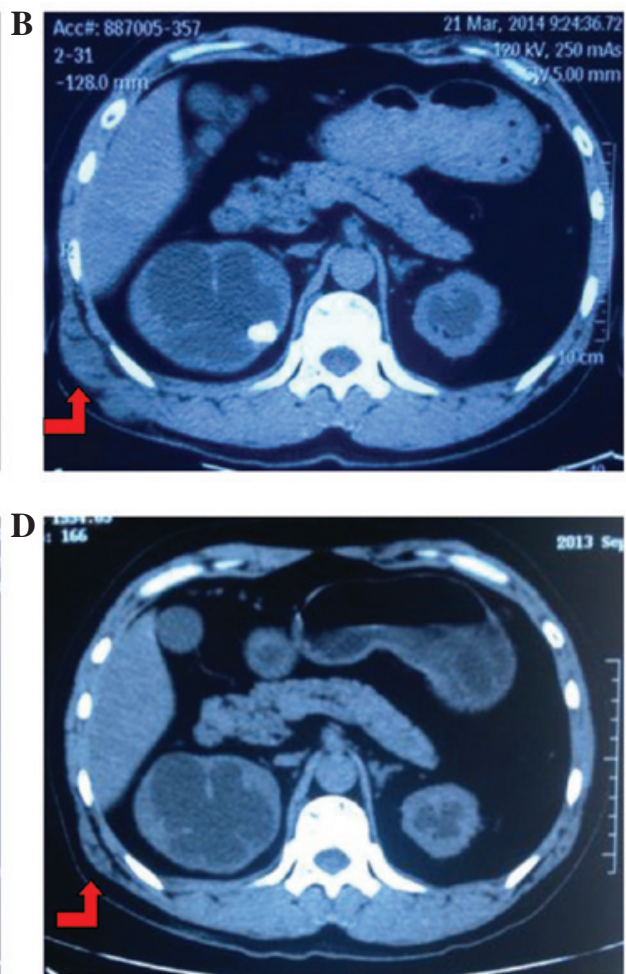

Figure 2. Images demonstrating a heterogeneous mass in the right flank. (A) Intravenous pyelogram, indicating poor functioning of the left kidney with atrophy and right moderate hydronephrosis with a large staghorn stones. (B) Computed tomography (CT) and (C) enhanced CT scan, revealing the soft tissue mass. (D) Positron emission tomography-CT scan, revealing radioactivity concentration on tumor site in right waist. Red arrows indicate the location of the tumor.
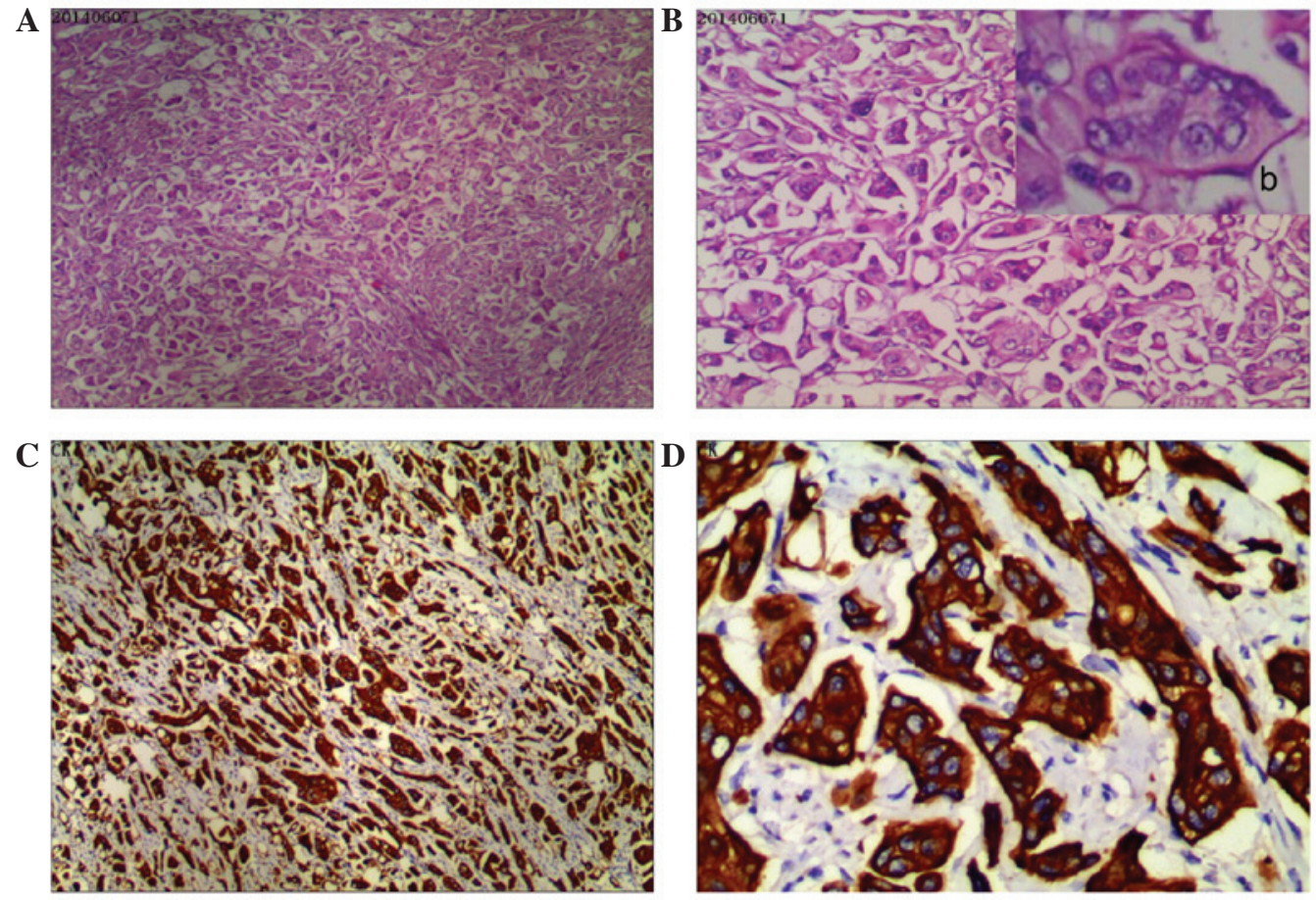

Figure 3. Histopathological examination revealing a poorly differentiated adenocarcinoma that conforms to neoplasm recurrence. (A and B) Hematoxylin and eosin staining visualized at (A) x40, (B) x100 and (Bb) x400 magnification. (C and D) Immunohistochemical staining demonstrating positivity for (C) cytokeratin and (D) epithelial membrane antigen (magnification, $\mathrm{x} 100$ ).

In 2011, it was estimated that 60,920 novel cases of RCC were diagnosed in the United States (10). A large number of RCC masses are diagnosed in asymptomatic patients in the early stages of the disease due to the increased application of cross-sectional imaging (11). However, this cross-sectional imaging cannot increase the diagnosis rates to manage small or occult renal masses. Previously, Mullins and Rodriguez (9) demonstrated that RCC may seed at a percutaneous biopsy 
tract. The risk of RCC seeding at a PCNL tract is low; however, upper tract transitional cell carcinoma (TCC) seeding is not uncommon. For example, various studies have reported TCC seeding in patients following percutaneous management of upper tract TCC, nephrostomy tube placement for obstructive uropathy and renal mass biopsy (12). Clinicians deciding whether to perform PCNL should consider that TCC tumors are typically considered to have a greater implantation rate following percutaneous manipulation compared with RCC tumors. In the current case, the patient did not present any abnormalities in the right kidney or change in micturition, excluding the recurrence of kidney stones. Furthermore, clinical and radiological observations did not identify any other primary site of disease or a direct extension of the tumor from the right kidney.

The patient in the present study was aged 39 years. The left kidney was poorly functioning with atrophy and right moderate hydronephrosis with large staghorn stones was observed. Although the risk of PCNL tract seeding with RCC is low, it was theorized with a high degree of probability that the origin of the tumor was RCC. However, RCC could not be identified in the right kidney. Considering the despondent state of the patient, chemotherapy was recommended following surgery. It remains unclear how a diagnosis of the origin of the tumor can be definitely determined and if such patients should be recommended for targeted therapies.

In conclusion, the present study is, to the best of our knowledge, the first contemporary report of PCNL tract seeding with RCC. The current study raises awareness of this rare complication. Measures such as careful examination of the renal tissue during PCNL surgery must be taken to minimize the risk of developing RCC at the time of PCNL, to prevent clinicians from being discouraged from performing PCNL for the treatment of renal stones when required.

\section{Acknowledgements}

This study was supported by the Medjaden Academy \& Research Foundation for Young Scientists (grant no. MJR20150025) and supported by the China Medical Foundation (grant no. 313.2238).

\section{References}

1. Sivalingam S, Cannon ST and Nakada SY: Current practices in percutaneous nephrolithotomy among endourologists. J Endourol 28: 524-527, 2014.

2. Wong KA, Sahai A, Patel A, Thomas K, Bultitude M and Glass J: Is percutaneous nephrolithotomy in solitary kidneys safe? Urology 82: 1013-1016, 2013.

3. Opondo D, Gravas S, Joyce A, et al: Standardization of patient outcomes reporting in percutaneous nephrolithotomy. J Endourol 28: 767-774, 2014.

4. Pérez-Fentes DA, Gude F, Blanco B and Freire CG: Percutaneous nephrolithotomy: short and long term effects on health related quality of life. J Endourol 29: 13-17, 2015.

5. Kreydin EI and Eisner BH: Risk factors for sepsis after percutaneous renal stone surgery. Nat Rev Urol 10: 598-605, 2013.

6. Wang SS, Ho HC, Su CK, Chen WM, Cheng CL and Yang CR: Seeding of malignant renal tumor through a nephrostomy tract. J Chin Med Assoc 67: 308-310, 2004.

7. Huang A, Low RK and deVere White R: Nephrostomy tract tumor seeding following percutaneous manipulation of a ureteral carcinoma. J Urol 153: 1041-1042, 1995. Fernström I and Johansson B: Percutaneous pyelolithotomy. A new extraction technique. Scand J Urol Nephrol 10: 257-259, 1976.

8. Preminger GM, Assimos DG, Lingeman JE, Nakada SY, Pearle MS and Wolf JS Jr; AUA Nephrolithiasis Guideline Panel: Chapter 1: AUA guideline on management of staghorn calculi: diagnosis and treatment recommendations. J Urol 173: 1991-2000, 2005.

9. Michel MS, Trojan L and Rassweiler JJ: Complications in percutaneous nephrolithotomy. Eur Urol 51: 899-906, 2007.

10. Mullins JK and Rodriguez R: Renal cell carcinoma seeding of a percutaneous biopsy tract. Can Urol Assoc J 7: E176-E179, 2013.

11. Siegel R, Ward E, Brawley O and Jemal A: Cancer statistics, 2011: the impact of eliminating socioeconomic and racial disparities on premature cancer deaths. CA Cancer J Clin 61: 212-236, 2011.

12. Kane CJ, Mallin K, Ritchey J, Cooperberg MR and Carroll PR: Renal cell cancer stage migration: analysis of the National Cancer Data Base. Cancer 113: 78-83, 2008. 